\title{
NUMERICAL SIMULATION \\ OF DEFLAGRATION-TO-DETONATION TRANSITION BY COUPLED FLAME TRACKING - PARTICLE METHOD
}

\author{
S. M. Frolov and V.S. Ivanov \\ N. N. Semenov Institute of Chemical Physics \\ Russian Academy of Sciences \\ Moscow, Russia
}

\begin{abstract}
The objective of the study outlined in this paper was to develop the computationally efficient algorithm for multidimensional numerical simulation of deflagration-to-detonation transition (DDT) in gas-fueled airbreathing pulse detonation engine (PDE). It is implied that the availability of such an algorithm will allow for more realistic estimates of PDE performances (specific impulse, thrust, etc.) than those obtained with the presumption of direct detonation initiation. The new algorithm is based on the coupled Flame Tracking-Particle (FTP) method implemented into the standard Computational Fluid Dynamics (CFD) code solving the Reynolds Averaged Navier-Stokes equations by the control-volume technique. The coupled methodology has been applied to the two-dimensional (2D) numerical simulation of repeatable DDT in a propane-fueled PDE at Mach 3.0 flight conditions at altitudes 9.3 and $16 \mathrm{~km}$. The fuel-based specific impulse was estimated as $1700-1800 \mathrm{~s}$. The DDT was shown to be a feasible approach for practical PDEs.
\end{abstract}

\section{INTRODUCTION}

Realistic estimates of PDE performances (specific impulse, thrust, etc.) require consideration of DDT rather than direct detonation initiation in the PDE tube. Despite the DDT phenomenology is currently well understood [1], its adequate numerical simulation is still not possible. The reason is the necessity of resolving a wide spectrum of length and time scales inherent in the DDT phenomenon and, therefore, the need in extremely high CPU power.

The classic mechanism of DDT in a straight smooth tube includes several stages $[2,3]$, namely, $(i)$ forced mixture ignition with the formation of a laminar flame; (ii) progressing increase in the rate of combustion because of the

This is an Open Access article distributed under the terms of the Creative Commons Attribution-Noncommercial License 3.0, which permits unrestricted use, distribution, and reproduction in any noncommercial medium, provided the original work is properly cited. 
incipience of instabilities and subsequently turbulent flow ahead of the flame front; (iii) shock wave formation and strengthening ahead of the accelerating flame front; and $(i \nu)$ self-ignition of the shock-compressed mixture in the region between the shock wave and flame front [4] ("explosion in the explosion" [5]) resulting in the formation of an overdriven detonation wave, and then $(\nu)$ selfsustaining Chapman-Jouguet (CJ) detonation. The time and distance of the DDT are known to be largely determined by the first three stages [6]. Detonation in air mixtures of hydrocarbon fuels requires that the "visible" velocity of the turbulent flame front in the laboratory coordinate system is higher than about $1000 \mathrm{~m} / \mathrm{s}$ [7]. At such a flame front velocity, the shock wave running ahead has a velocity higher than $1300 \mathrm{~m} / \mathrm{s}$ (the shock wave Mach number is $\mathrm{M} \approx 3.8$ ), and the pressure and temperature of the explosive mixture behind it are higher than $1.7 \mathrm{MPa}$ and $1200 \mathrm{~K}$, respectively.

The mechanism of DDT in tubes with regular obstacles $[3,8]$ is in many respects similar to the mechanism [2-6] described above. There are also important differences. First, the flame is accelerated much more rapidly in a tube with obstacles because of obstacle-induced turbulence generation in the fresh explosive mixture. Second, new possibilities for gas ignition appear: the gas can ignite in a shock wave reflected from an obstacle or (if obstacles are large) due to mixing of jets of hot combustion products with the cold explosive mixture.

At present, there exist few attempts of numerical simulation of DDT in gases. The most successful is seemingly one by Oran and Gamezo who published a series of papers on DDT in hydrogen-air mixtures in channels with regular obstacles $[9,10]$. They solved both three-dimensional (3D) and 2D Navier-Stokes equations coupled with the energy conservation equation and the kinetic equation for the single-stage overall chemical reaction. Despite the results of $[9,10]$ revealed some salient features of flame acceleration in the obstructed channels, the model applied in $[9,10]$ cannot be used for quantitative predictions. This model does not make a difference between the chemical kinetics of combustion in the flame front and chemical kinetics of self-ignition. As is well known, the reactions in flame do not exhibit ignition delays as the reactive mixture is ignited due to diffusion of heat and active species from the high-temperature reaction zone. At self-ignition, neither heat nor active species are supplied from outside and, therefore, the initial increase of the reaction rate is significantly slower than in the flame and the main role is played by relatively slow chain origination reactions. The latter means that the reaction rate constant in the overall reaction mechanism describing preflame self-ignition should be significantly lower than that describing the reaction in the flame front. As a result, the use of identical reaction rate constants for both preflame and flame reactions in $[9,10]$ can become a reason of significant overestimation of preflame reaction rates.

The objective of any combustion model is to provide correct values of mean reaction rates regardless the combustion mode in a turbulent reactive flow. The 
mean reaction rate can be obtained provided one knows the reaction kinetics and the local instantaneous fields of temperature and species concentrations. The development of reaction kinetics is the separate task which is independent of combustion modeling. The only relevant issue in this respect is the CPU time required for calculating instantaneous reaction rates. This issue can be overcome by applying properly validated short overall reaction mechanisms and/or look-up tables. The local instantaneous fields of temperature and species concentrations are usually not known. Therefore, one has to replace this lacking information by a combustion model.

There exist many combustion models both for laminar and turbulent flows. If chemistry is fast as compared to mixing, the Eddy-Break-Up model can be used [11]. It is simple but has a limited range of validity. There is a whole class of statistical combustion models (based on the formalism of probability density functions) with probabilistic representation of turbulence and its interaction with chemistry [12]. Despite many attractive features, this approach is still not capable of operating with complex chemistry due to large CPU requirements. Moreover, such approaches do not resolve different scales in the turbulent flow and their energy content. Instead, all scales are treated indifferently whereas their effect on combustion and flame is different. Nevertheless, these approaches look very promising for treating self-ignition problems.

The other class of models deals with the flamelet approach [13]. In this approach, the instantaneous flame is assumed to consist of localized reactive sheets, which are transported by the flow and wrinkled by turbulent eddies. The flamelet approach is applicable when the characteristic turbulent scales are larger than the typical flame thickness. This condition is satisfied in many practical situations. The flamelet models are usually based on the flame surface density concept or apply probability density functions. One of the most attractive flamelet models is based on the balance equation for the flame surface density. This equation governs the transport of the mean reactive surface by the turbulent flow and includes physical mechanisms responsible for flame surface area production and destruction.

The approach used within this study is also based on considering the flame surface area. However, to speed up calculations, instead of solving the partial differential equation for the flame surface density, it implies explicit tracing of the mean reactive surface and application of the laminar/turbulent flame velocity concepts.

\section{FLAME TRACKING METHOD}

The approach outlined below will be referred to as the model of laminar/ turbulent combustion. Let first explain the essence of the model on the example 


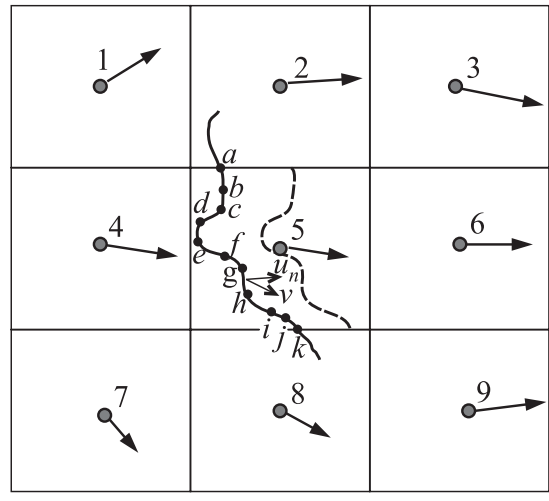

Figure 1 Laminar flame model

of laminar flame propagation. The flame surface shape and area can be found based on the Huygens principle (Fig. 1): Each elementary portion of the flame surface $a-b-c-\ldots-i-j-k$ displaces in time due to burning of the fresh mixture at local velocity $u_{n}$ (normal to the flame surface) and due to convective motion of the mixture at local velocity $v$ (see segment $g-h$ ). The local instantaneous velocity $u_{n}$ can be taken from the look-up tables including in general the effects of mixture dilution with combustion products, flame stretching, and flammability limits. The local instantaneous velocity $v$ can be calculated using a high-order interpolation technique (e.g., using the velocity values in nodes 1 to 9 in Fig. 1). In Fig. 1, the flame surface is represented by straight line segments. In 3D calculations, the flame surface will be represented by connected triangles.

The energy release rate in the computational cell, $\dot{Q}$, is composed of two terms: energy release due to frontal combustion, $\dot{Q}_{f}$, and energy release due to volumetric reactions, $\dot{Q}_{v}$. The first term $\dot{Q}_{f}$ can be calculated based on the estimated instantaneous flame surface area $S_{n}$ and the laminar flame propagation velocity $u_{n}$ :

$$
\dot{Q}_{f}=\rho Q \sum S_{n i} u_{n i}
$$

where $\rho$ is the density of the reactive mixture, $Q$ is the combustion heat, and summation is made over all flame segments (e.g., segment $g-h$ in Fig. 1) in the cell. The second term $\dot{Q}_{v}$ can be calculated using the Particle method (see section 3).

In the turbulent flow field, a pulsating velocity vector distorts the "mean" reactive (flame) surface as shown schematically in Fig. 2 by the thin wrinkled solid curve. The local instantaneous flame wrinkling can be taken into account by proper increasing the normal flame velocity, or in other words, by introducing a concept of local turbulent flame velocity $u_{T}$. The local turbulent flame velocity (e. g., at segment $g-h$ in Fig. 2) is defined as

$$
u_{T}=\frac{u_{n} S}{S_{T}}
$$

where $S$ is the surface area of the wrinkled flame at a given segment and $S_{T}$ is the surface area of the equivalent "planar" flame (straight line $g-h$ in Fig. 2). 
The problem now is to find the way of calculating $u_{T}$. In the theory of turbulent combustion, there are many correlations between $u_{T}$ and $u_{n}$. One of the classical correlations is Shchelkin formula [14]:

$$
u_{T} \approx u_{n} \sqrt{1+\frac{u^{\prime 2}}{u_{n}^{2}}}
$$

where $u^{\prime}$ is the local turbulence intensity related to the turbulent kinetic energy or to pulsating velocity correlations. Instead of Eq. (1), one can use other available corre-

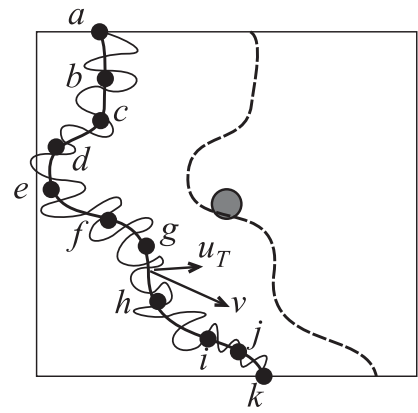

Figure 2 Turbulent flame model lations for the turbulent flame velocity.

One can apply the Huygens principle to model only the "mean" shape of the turbulent flame (solid curve $a-b-c-d . .-i-j-k$ in Fig. 2): each elementary portion of the flame surface $a-b-c-\ldots-i-j-k$ displaces in time due to burning of the fresh mixture at local velocity $u_{T}$ (normal to the flame surface) and to convective motion of the mixture at local velocity $v$ (see segment $g-h$ ). The new "mean" flame position is shown by the thick dashed curve in Fig. 2.

The mean energy release rate in the cell, $\dot{Q}$, is composed of two terms: energy release due to frontal combustion, $\dot{Q}_{f}$, and energy release due to volumetric combustion, $Q_{v}$. The first term $\dot{Q}_{f}$ can be calculated based on the estimated "mean" flame surface area and the turbulent flame propagation velocity using Eq. (1) (or other correlations). Equation (1) relates the turbulent flame velocity to laminar flame velocity (tabulated) and local turbulence intensity (provided by any model of turbulence). Based on such an equation, one can calculate the mean (frontal) energy release rate in the computational cell as the sum

$$
\dot{Q}_{f}=\rho Q \sum S_{T i} u_{T i}
$$

where index $i$ relates to the flame surface segment (e.g., $g-h$ in Fig. 2). The second term $\dot{Q}_{v}$ can be calculated using the Particle method (see section 3 ).

Thus, the model of turbulent premixed combustion does not differ much from that of the laminar premixed combustion, except for using $u_{T}$ instead of $u_{n}$. Moreover, the formulae like Eq. (1) are asymptotically valid for the model of laminar combustion (when $u^{\prime} \rightarrow 0, u_{T} \rightarrow u_{n}$ ). It stands to reason that the turbulent combustion model will be also valid for the laminar combustion as a limiting case. This is one of the model advantages. This feature will allow using the same model to calculate the initial laminar flame kernel growth from the spark ignition with continuous transition to turbulent combustion. The combustion model under consideration does not contain tuning parameters (some parameters can be introduced with the equations replacing Eq. (1)). This is the 
other important model advantage. The additional advantage of the model is that, when coupled with the Particle method, it will cover both possible modes of premixed combustion, namely, frontal and volumetric.

It is expected that the accuracy of the computational results will be mainly affected by the turbulence model used. The main problem in implementing such a combustion model into a CFD code is the development of an efficient algorithm for "mean" flame-shape tracing inside computational cells. This algorithm should meet the constraints on the flame-front continuity, connectivity, etc., and the constraints on the CPU time consumption.

\section{PARTICLE METHOD}

The Particle method allows continuous monitoring of preflame and postflame reactions using the kinetic database [15]. The preflame zone exhibits volumetric reactions of fuel oxidation, formation of intermediate products like alcohols, aldehydes, peroxides, etc. In general, the preflame reactions are inhomogeneous due to inhomogeneous distributions of temperature and main species concentrations. The preflame reactions can result in the localized energy release. For example, low-temperature cool-flame oxidation of $n$-alkane fuels can result in the release of up to $10 \%-15 \%$ of the total reaction heat. Thus, in general, two-way coupling approach has to be applied for the preflame reactions; however, in some cases, one-way coupling is also possible. The direct (and CPU time consuming) way to calculate the volumetric reaction rates is to solve the equations of chemical kinetics in the preflame zone in each computational cell with due regard for turbulent fluctuations of temperature and species concentrations. To shorten the CPU time, one can introduce a certain number of trace Lagrangian particles which will move in the preflame zone according to the local instantaneous velocity vector. In each particle, the preflame reactions will proceed at the rates determined by its instantaneous temperature and species concentrations. For determining the time and location of preflame self-ignition, there will be a need in adopting a certain criterion. Such a criterion can be based on the fixed rate of temperature rise in the particle, e.g., $10^{6}$ or $10^{7} \mathrm{~K} / \mathrm{s}$.

In case of two-way coupling, the reaction rate is calculated in each particle based on its temperature and species concentrations, and the mean (over all particles in cell) reaction rate directly affects the mean flow pattern. When a self-ignition criterion is met in one or several particles, new (forced) ignition sites in the preflame zone can be automatically introduced. In general, these ignition sites give birth to new laminar/turbulent flame kernels or, if the preflame reactions are fast, they result in the induction (spontaneous) flames and volumetric combustion. For keeping the number of particles at a reasonable level, the consistent procedures of particle cloning and clustering should be developed. 
The preflame particles are traced until the entire geometry is traversed by the frontal or volumetric combustion.

In each $i$ th Lagrangian particle, the following set of equations is solved [15]:

- equation of motion:

$$
\frac{d x_{k}^{i}}{d t}=u_{k}^{i}
$$

where $x_{k}^{i}$ is the Cartesian coordinate $(k=1,2$, and 3$)$ and $u_{k}^{i}$ is the corresponding velocity component;

- mass conservation equation:

$$
\frac{d\left(\rho_{l}^{i} V^{i}\right)}{d t}=\nabla J_{l}^{i}+J_{h l}^{i}
$$

where $\rho_{l}^{i}$ is the partial density of the $l$ th species, $V^{i}$ is the particle volume, $\nabla J_{l}^{i}$ is the diffusion flux of the $l$ th species to/from the particle, and $J_{h l}^{i}$ is the flux of the $l$ th species due to chemical reaction;

- momentum conservation equation:

$$
\rho^{i} \frac{d u_{k}^{i}}{d t}=\frac{\partial P^{i}}{\partial x_{k}}-\nabla\left(I p^{i}-\tau^{i}\right)
$$

where $\rho^{i}$ is the mean particle density $\left(\rho^{i}=\sum \rho_{l}^{i}\right) ; P^{i}$ is the mean pressure; $p^{i}$ is the pulsating pressure; $I$ is the unit tensor; and $\tau^{i}$ is the molecular viscous stress; and

- energy conservation equation:

$$
\rho^{i} \frac{d h^{i}}{d t}=\nabla q^{i}+h_{\mathrm{hom}}^{i}+\frac{\partial P^{i}}{\partial t}-P^{i} \frac{\partial u_{k}^{i}}{\partial x_{k}}
$$

where $h^{i}$ is the particle enthalpy; $q^{i}$ is the heat flux to/from the particle; and $h_{\mathrm{hom}}^{i}$ is the rate of heat release due to chemical reaction. The volumetric term $\dot{Q}_{v}$ mentioned above (see section 2) is calculated based on $h_{\mathrm{hom}}^{i}$ as

$$
\dot{Q}_{v}=\sum h_{\mathrm{hom}}^{i} V_{i}
$$

Molecular diffusion term $\nabla J_{l}^{i}$ in Eq. (2), molecular heat transfer term $\nabla q^{i}$ in Eq. (4), and term $\nabla\left(I p^{i}+\tau^{i}\right)$ in Eq. (3) require modeling. These terms are modeled using classical models of Interaction by Exchange with the Mean [16]:

- diffusion term:

$$
\nabla J_{l}^{i}=-0.5 C_{1}\left(Y_{l}^{i}-\bar{Y}_{l}^{i}\right) \rho^{i} V^{i} \omega
$$


where $C_{1}$ is the coefficient $\left(C_{1} \approx 2.0\right) ; Y_{l}^{i}$ is the concentration of the $l$ th species; $\bar{Y}_{l}^{i}$ is the mean concentration of the $l$ th species at the location of the $i$ th particle; and $\omega$ is the turbulent frequency;

- heat exchange term:

$$
\nabla q^{i}=-0.5 C_{2}\left(h^{i}-\bar{h}^{i}\right) \rho^{i} \omega
$$

where $C_{2}$ is the coefficient $\left(C_{2} \approx 2.0\right) ; h^{i}$ is the enthalpy; and $\bar{h}^{i}$ is the mean enthalpy in the location of the $i$ th particle;

- term $\nabla\left(I p^{i}-\tau^{i}\right)$ :

$$
\left(\rho^{i}\right)^{-1} \nabla\left(p^{i} I-\tau^{i}\right)=-\zeta\left(u_{k}^{i}-\bar{u}_{k}^{i}\right)+A(t)
$$

where $\bar{u}_{k}^{i}$ is the mean velocity at the location of the $i$ th particle; $\zeta$ is the coefficient $(\zeta \approx 2.075 \omega)$; and $A(t)$ is the stochastic function in the Langevin equation [16].

For effective implementation of the Particle method, the reaction rate term $J_{h l}^{i}$ in Eq. (2) and heat release term $h_{\text {hom }}^{i}$ in Eq. (4) can be neglected in those preflame domains where the local temperature is less than a certain conditional value $T_{c}$. This assumption allows omitting routine chemical kinetic calculations in the major part of the preflame zone with $T<T_{c}$. Thus, each computational cell contains a certain number $N$ of particles with $N$ chosen to support a proper statistical accuracy. The preflame domain is then represented by stochastically moving "cold" (with $T^{i}<T_{c}$ ) and "hot" (with $T^{i}>T_{c}$ ) Lagrangian particles. In long tubes with one open end (like a PDE tube), most of particles are "cold" and routine kinetic calculations for them are not required. "Hot" particles are concentrated in the region between the flame-induced shock wave and the flame itself.

\section{KINETIC DATABASE}

The coupled FTP algorithm is supplemented with the database of tabulated laminar flame velocities for the mixtures of $n$-alkane fuels (methane, propane, etc.) with air in the wide range of fuel-air ratio (from fuel-lean to fuel-rich flammability limits), initial temperature (up to $900 \mathrm{~K}$ ), and pressure (up to $10 \mathrm{MPa}$ ) as well as the reaction kinetics of fuel self-ignition [15]. As an example, Table 1 shows the look-up table for the laminar flame velocity and Tables 2 and 3 show the overall reaction mechanism of preflame oxidation in the stoichiometric propane-air mixture. 
Table 1 Laminar flame velocities for the stoichiometric propane-air mixture, $\mathrm{cm} / \mathrm{s}$

\begin{tabular}{ccrrrr}
\hline \multirow{2}{*}{ Pressure, MPa } & \multicolumn{5}{c}{ Temperature, K } \\
\cline { 2 - 6 } & 300 & 450 & 600 & \multicolumn{1}{c}{750} & 900 \\
\hline 0.1 & 39 & 78 & 143 & 247 & 451 \\
0.3 & 28 & 55 & 102 & 178 & 306 \\
1.0 & 19 & 35 & 64 & 112 & 191 \\
4.0 & 8.9 & 19 & 36 & 62 & 105 \\
10.0 & 6.0 & 13 & 24 & 41 & 69 \\
\hline
\end{tabular}

Table 2 Reaction mechanism of preflame propane oxidation

\begin{tabular}{|c|c|c|c|c|c|}
\hline \multirow[b]{2}{*}{ Reaction } & \multirow{2}{*}{$\begin{array}{c}\mathrm{H}, \\
\mathrm{kcal} / \mathrm{mol}\end{array}$} & \multicolumn{2}{|c|}{ Forward } & \multicolumn{2}{|c|}{ Reverse } \\
\hline & & $\begin{array}{c}A, \\
\mathrm{~mol}, \mathrm{l}, \mathrm{s}\end{array}$ & $\begin{array}{c}E, \\
\mathrm{kcal} / \mathrm{mol}\end{array}$ & $\begin{array}{c}A, \\
\mathrm{~mol}, \mathrm{l}, \mathrm{s}\end{array}$ & $\begin{array}{c}E, \\
\mathrm{kcal} / \mathrm{mol}\end{array}$ \\
\hline 1. $\mathrm{C}_{3} \mathrm{H}_{8}+3.5 \mathrm{O}_{2} \rightarrow 3 \mathrm{CO}+4 \mathrm{H}_{2} \mathrm{O}$ & 290.0 & $A_{1}$ & $E_{1}$ & & \\
\hline 2. $\mathrm{CO}+\mathrm{H}_{2} \mathrm{O} \leftrightarrow \mathrm{CO}_{2}+\mathrm{H}_{2}$ & 10.0 & $\frac{1.0 \cdot 10^{12}}{P}$ & 41.5 & $\frac{3.1 \cdot 10^{13}}{P}$ & 49.1 \\
\hline 3. $\mathrm{H}_{2}+\mathrm{H}_{2}+\mathrm{O}_{2} \rightarrow \mathrm{H}_{2} \mathrm{O}+\mathrm{H}_{2} \mathrm{O}$ & 114.0 & $\frac{7.0 \cdot 10^{13}}{P^{0.5}}$ & 21.0 & & \\
\hline 4. $\mathrm{CO}+\mathrm{CO}+\mathrm{O}_{2} \rightarrow \mathrm{CO}_{2}+\mathrm{CO}_{2}$ & 134.0 & $\frac{8.5 \cdot 10^{12}}{P^{1.5}}$ & 21.0 & & \\
\hline
\end{tabular}

Remarks: (1) reaction rate constant is defined as $k=A T \exp (-E /(R T))$; (2) reaction 1 is considered as bimolecular; (3) pressure is taken in atmospheres.

Table 3 Kinetic parameters of rate limiting reaction 1

\begin{tabular}{rcc}
\hline \multirow{2}{*}{$P, \operatorname{atm}$} & \multicolumn{2}{c}{$A_{1}, \mathrm{l} /(\mathrm{mol} \cdot \mathrm{s})$} \\
\cline { 2 - 3 } & $T<775 \mathrm{~K}, E_{1}=40 \mathrm{kcal} / \mathrm{mol}$ & $T>775 \mathrm{~K}, E_{1}=45 \mathrm{kcal} / \mathrm{mol}$ \\
\hline 1 & $19.60 \cdot 10^{11} \mathrm{P}$ & $17.30 \cdot 10^{11} / P^{0.23}$ \\
20 & $19.60 \cdot 10^{11} P$ & $8.77 \cdot 10^{11} / P^{0.23}$ \\
40 & $19.60 \cdot 10^{11} P$ & $7.50 \cdot 10^{11} / P^{0.23}$ \\
80 & $19.60 \cdot 10^{11} P$ & $6.41 \cdot 10^{11} / P^{0.23}$ \\
120 & $1.96 \cdot 10^{11} P$ & $5.85 \cdot 10^{11} / P^{0.23}$ \\
\hline
\end{tabular}

Note that the reaction mechanism of Tables 2 and 3 describes a two-stage low-temperature self-ignition of propane at $T<775 \mathrm{~K}$ [17]. In this case, the temperature history of the mixture in an adiabatic reactor exhibits two stages: "cool flame" with the induction period $\tau_{1}$ and subsequent "hot explosion" with the induction period $\tau_{2}$. The overall induction period is $\tau=\tau_{1}+\tau_{2}$. To obtain such a behavior of the temperature curve based on Tables 2 and 3, one has to use two different sets of kinetic parameters for the rate limiting reaction 1: one at $T<775 \mathrm{~K}$ and another at $T>775 \mathrm{~K}$ (see Table 3 ). 


\section{RESULTS OF TEST CALCULATIONS}

The coupled FTP method has been developed and implemented into the standard CFD code (here, AVL FIRE). Before applying the FTP method to the PDE geometry, it was extensively validated against available experimental data. Presented below are some validation examples.

\subsection{Flame Acceleration in a Long Smooth-Walled Channel}

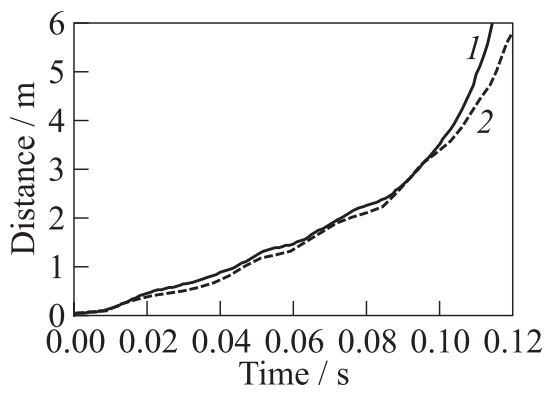

Figure 3 Comparison between predicted (1) and measured [18] (2) distance traveled by the flame vs. time in $40 \times 40 \mathrm{~mm}$ straight smooth-walled channel $6.1 \mathrm{~m}$ long filled with the stoichiometric propane-air mixture at $T_{0}=293 \mathrm{~K}$ and $P_{0}=1 \mathrm{~atm}$
Consider the 2D test case with flame acceleration in the straight rectangular $40 \times 40 \mathrm{~mm}$ channel $6.1 \mathrm{~m}$ long filled with the stoichiometric propane-air mixture at normal initial conditions as used in experiments [18]. In the experiments, the mixture was initially quiescent and ignition took place at approximately $1 \mathrm{~cm}$ from the closed end of the channel.

Figure 3 compares the results of calculation with the experiment in terms of the time history of the distance traveled by the flame. Curve 1 corresponds to the predicted results whereas curve 2 corresponds to the measurements. The walls of the channel were assumed isothermal $\left(T_{w}=293 \mathrm{~K}\right)$. At the open end, a constant-pressure $\left(P_{0}=0.1 \mathrm{MPa}\right)$ boundary condition was applied. The use of nonreflecting boundary conditions [19] at the walls of a buffer volume of a larger cross section attached to the open end of the channel did not affect significantly the results of calculations leading, however, to increasing CPU time. The stoichiometric propane-air mixture was assumed initially quiescent at $T_{0}=293 \mathrm{~K}$ and $P_{0}=0.1 \mathrm{MPa}$. The initial flame kernel was taken as a circle $1 \mathrm{~mm}$ in radius with the center located at $1 \mathrm{~cm}$ from the closed end-wall at the symmetry plane. The turbulent flame velocity was modeled by Eq. (1). The laminar flame velocity entering Eq. (1) was linearly interpolated using the data of lookup tables. Turbulence was modeled by the standard $k-\varepsilon$ model. The flame front in a computational cell was normally represented by no less than 15 segments. As seen from Fig. 3, the predicted flame front trajectory agrees well with the measurements despite the $2 \mathrm{D}$ representation of essentially $3 \mathrm{D}$ phenomena in the 
experiment. It is worth noting that the numerical simulation is capable of adequate predicting the effect of various pressure waves on flame motion which is obvious from simultaneous appearance of crests on the curves.

\subsection{Flame Acceleration in a Tube with Regular Obstacles}

Consider the results of $2 \mathrm{D}$ test cases with flame acceleration in the straight cylindrical tube of internal diameter $D=152 \mathrm{~mm}$ and length $L=3.1 \mathrm{~m}$ with regular obstacles in the form of orifice plates with blockage ratios 0.43 , 0.6 , and 0.75 filled with the stoichiometric propane-air mixture at normal initial conditions as used in experiments [20]. The blockage ratio of orifice plates was defined as $\mathrm{BR}=1-(d / D)^{2}$ where $d$ is the orifice diameter. In the experiments, the mixture was initially quiescent and spark ignition took place at the closed end of the tube. The tube was represented by a cylinder segment with the structured computational grid. All settings were the same as in the previous example.

Figure 4 compares predicted visible flame velocities (curves) with experimental data [20] (symbols) depending on the distance traveled by the flame for the three different values of $B R$. In all cases, the distance between neighboring orifice plates (pitch $S$ ) was equal to tube diameter $S=D$.

In general, satisfactory agreement between predicted and mea-

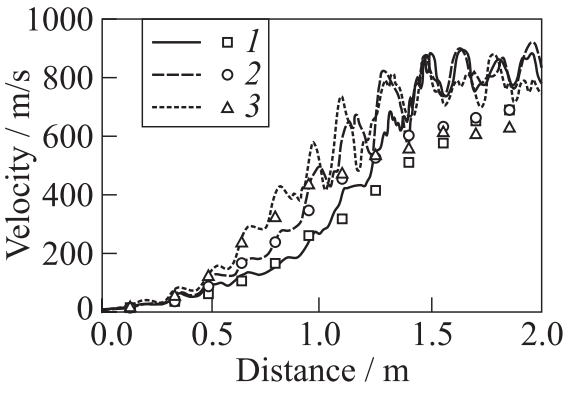

Figure 4 Predicted (curves) and measured (symbols [20]) visible flame velocities vs. distance in tubes of $D=152 \mathrm{~mm}$ with regular orifice plates $(\mathrm{BR}=0.43(1), 0.6(2)$, and $0.75(3)$, pitch $S=D)$. Stoichometric propane-air mixture at $T_{0}=293 \mathrm{~K}$ and $P_{0}=1 \mathrm{~atm}$ sured values of flame velocity is obtained. Very good agreement exists at the initial phase of flame acceleration, where its velocity is less than $300-400 \mathrm{~m} / \mathrm{s}$. The flame accelerates to about 800 $900 \mathrm{~m} / \mathrm{s}$, i. e., to the velocity close to the sound speed in the combustion products (about $890 \mathrm{~m} / \mathrm{s}$ ). Initially, the flame accelerates faster in the tube with orifice plates of larger BR. This is probably caused by a higher level of turbulence generated by such obstacles. However, further flame acceleration results in growing momentum losses and the efficiency of orifice plates with high BR in terms of flame acceleration decreases. The important finding from the calculations is that the flame velocity grows in time with oscillations rather than monotonically as could be thought based on the experimental points. 


\subsection{Flame Acceleration in a Complex Geometry Duct}

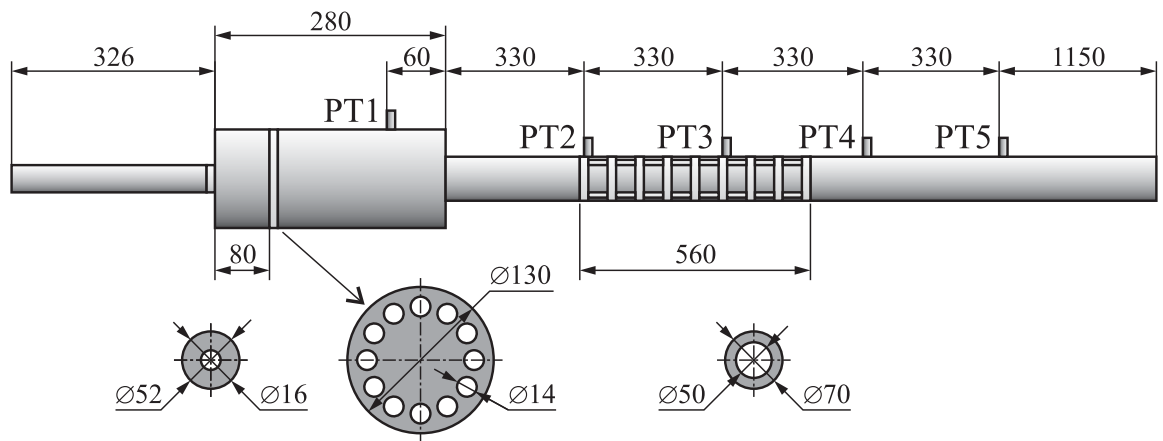

Figure 5 Schematic of experimental setup. Dimensions are in millimeters

The performance of the FTP method was also validated for the acceleration of stoichiometric methane-air flame in the complex-geometry duct shown in Fig. 5 with a prechamber $326 \mathrm{~mm}$ long, expansion volume $280 \mathrm{~mm}$ long, and a tube $2470 \mathrm{~mm}$ long with orifice plates and perforated partitions as used in the experiments performed recently at Semenov Institute of Chemical Physics (SICP) [21]. In the experiments, the mixture was ignited by a spark plug at the left (closed) end of the prechamber. After traversing the prechamber, the accelerating turbu-

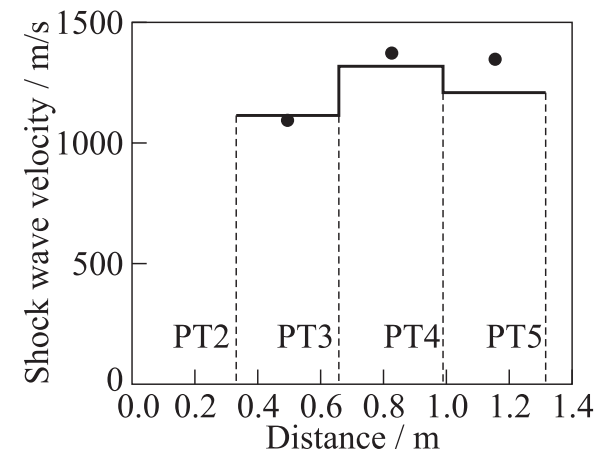

Figure 6 Comparison of predicted (line) and measured [21] (circles) shock wave velocities in the setup of Fig. 5. Height of circles corresponds to the error of velocity measurement. Distance is measured from the inlet to the 70-millimeter tube lent flame entered the expansion volume through a nozzle $16 \mathrm{~mm}$ in diameter. In the expansion volume, flame further accelerated when passing through the perforated partition with 12 orifices, each $14 \mathrm{~mm}$ in diameter. Thereafter, the flame transitioned to a 70-millimeter diameter tube with an array of regular obstacles in the form of orifice plates with the blockage ratio $\mathrm{BR}=0.5$ and spacing equal to tube diameter (70 mm). The far (right) end of the tube was open to the atmosphere.

In the calculations, the duct of Fig. 5 was represented by the axisymmetrical geometry. Instead of perforations, annular orifices were considered. The stoichiometric 
mehane-air mixture was assumed initially quiescent at $T_{0}=293 \mathrm{~K}$ and $P_{0}$ $=1$ atm. All other settings were the same as in the previous validation examples.

Figure 6 compares the predicted and measured mean shock wave velocity at three measuring segments PT2-PT3, PT3-PT4, and PT4-PT5, where PT stands for pressure transducer. Taking into account the complexity of setup geometry, the results of calculations can be treated as encouraging.

\subsection{Simulation of Preflame Self-Ignition}

The coupled FTP methodology was applied to the 2D numerical simulation of flame acceleration in a channel with flame accelerating obstacles filled with the stoichiometric propane-air mixture at normal initial conditions (Fig. 7). The channel was $40 \mathrm{~mm}$ wide with regular obstacles $2 \times 2 \mathrm{~mm}$ size installed with a pitch of $20 \mathrm{~mm}$ along the entire channel length. The boundary conditions and ignition were modeled in the same way as described above. In the calculations, only the upper part of the channel was considered with symmetry boundary conditions along the symmetry plane. For tracing possible self-ignition events, the preflame zone was represented by notional particles. The number of particles in each computational cell was controlled to be no less than 3 and no more than 12 with the mean value of 6 . The self-ignition of a particle was assumed to occur when the rate of temperature increase in this particle exceeded $10^{6} \mathrm{~K} / \mathrm{s}$. The two-way coupling procedure between the particles and the mean flow was used. The self-ignition of at least one particle in a cell was treated as the self-ignition of mixture in the cell. The channel configuration and the conditional treatment of preflame self-ignition were used to check the capability of the FTP method to predict the DDT phenomenon.

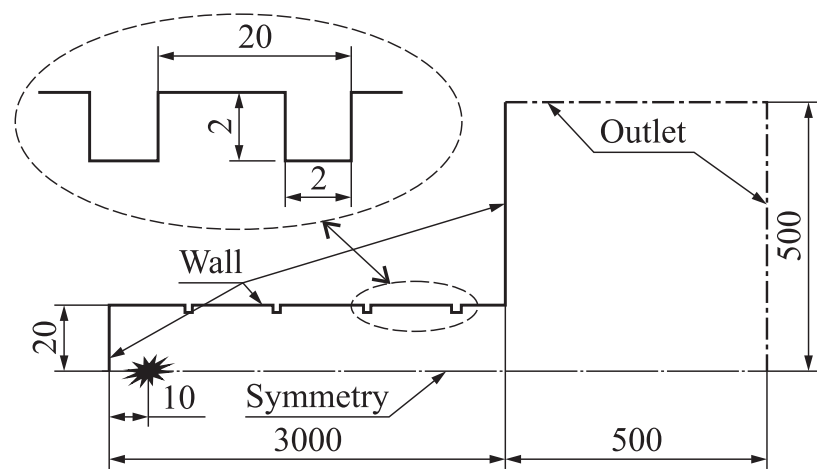

Figure 7 Schematic of an obstructed channel. Dimensions are in millimeters 


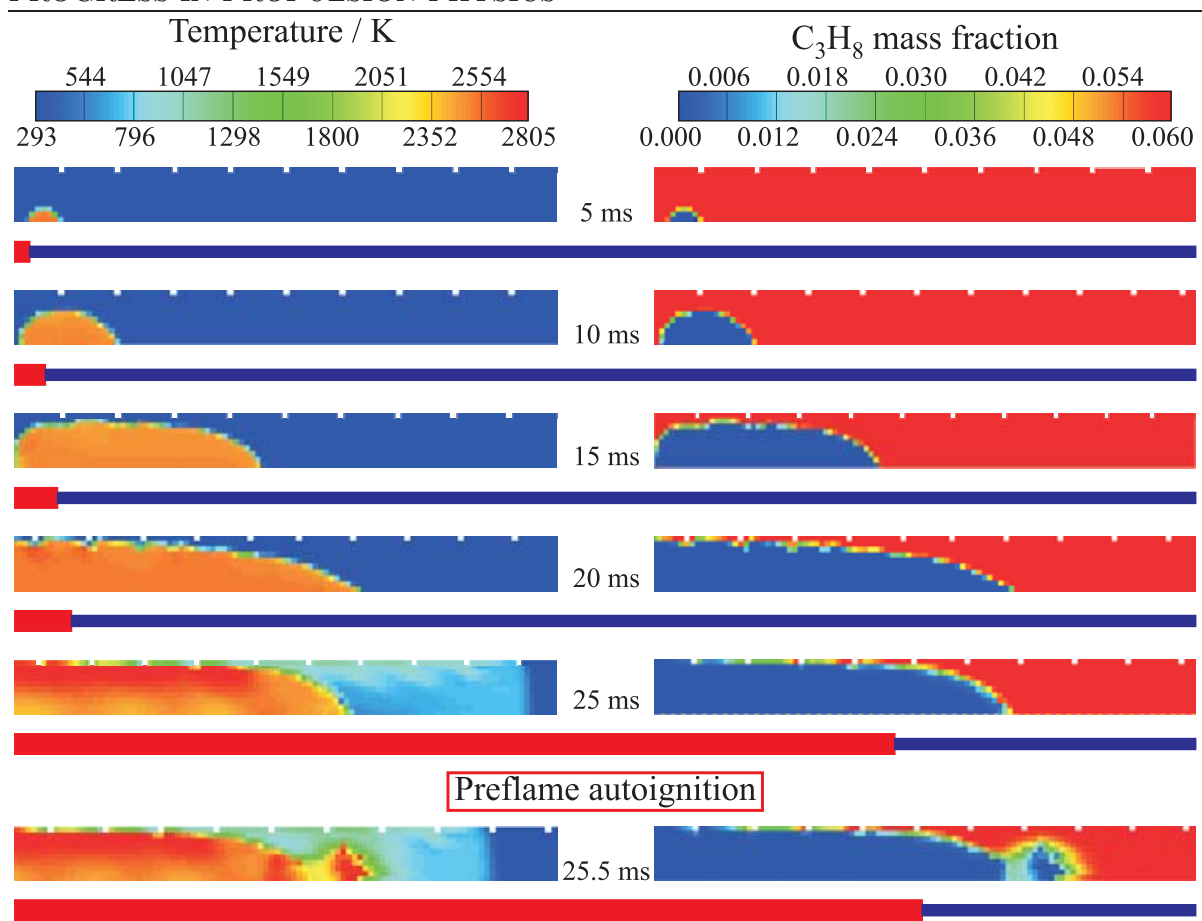

Figure 8 Predicted temperature (left) and propane mass fraction (right) fields at different time after ignition. The leading edges of red bars below the snapshots represent the instantaneous spatial positions of the flame front in the channel $3 \mathrm{~m}$ long. (Refer Frolov and Ivanov, p. 546.)

Figure 8 shows the predicted temperature and propane mass fraction fields at different time after ignition. The first five snapshots of temperature (left) and propane mass fraction (right) are plotted with a time interval of $5 \mathrm{~ms}$. These snapshots correspond to the relatively slow initial stage of flame acceleration preceding preflame self-ignition. The last snapshot of temperature and propane mass fraction is plotted for the time $25.5 \mathrm{~ms}$.

At the temperature snapshot corresponding to $25 \mathrm{~ms}$, one can clearly see the lead shock wave and bow shocks attached to the obstacles. The latter indicates that the postshock flow is supersonic. The instantaneous spatial temperature distributions in the preflame zone are nonuniform. At the snapshot corresponding to $25.5 \mathrm{~ms}$, preflame self-ignition occurs. One can clearly see the exothermic centers ("hot spots") in the preflame zone. The hot spots are located in the central part of the channel rather than at its periphery due to the cold wall boundary condition. Interestingly, the cluster of exothermic centers originates at a certain distance from the flame front rather than directly ahead of it. This is caused by the existence of bow shocks and rarefaction fans generated by obstacles in the supersonic flow behind the lead shock wave and by the history of temperature and species concentrations in the preflame zone. As can be seen from comparing 


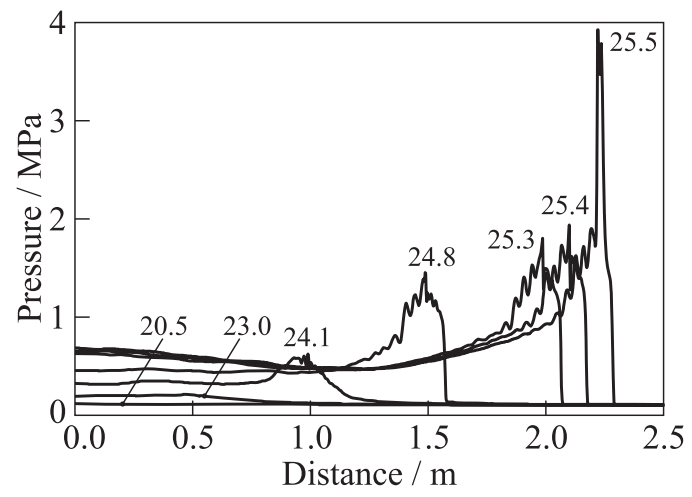

Figure 9 Predicted spatial pressure distributions in the channel at different time (in $\mathrm{ms}$ ) after ignition

the temperature snapshots at 25 and $25.5 \mathrm{~ms}$, preflame self-ignition occurs in the region compressed by the bow shock.

Contrary to the relatively slow initial stage of flame acceleration, the evolution of the DDT process after preflame self-ignition is very fast. In the presence of preflame self-ignition, two modes of combustion become possible simultaneously, namely, the frontal and volumetric. Remind that the frontal mode is calculated using the Flame Tracking method whereas the volumetric mode is calculated using the Particle method. Formally, for adequate simulation of the DDT process, one has to take into account the transition of the volumetric mode to the frontal one, in particular, in the relatively cold near-wall region. At this stage of code development, this transition is not taken into account. Despite this fact does not affect much the DDT run-up distance and time (the volumetric mode spreads very fast predominantly in the longitudinal direction), it can affect the completeness of combustion and, therefore, the detonation velocity and other parameters (pressure, etc.).

Figure 9 shows the predicted spatial pressure distributions in the channel at different time intervals after spark ignition. Pressure oscillations are caused by bow shocks and rarefaction waves generated by regular obstacles. The localized "explosion in the explosion" occurring at about $25.5 \mathrm{~ms}$ results in pressure rise up to $4 \mathrm{MPa}$ followed by spontaneous detonation initiation (not shown in Fig. 9).

\section{PULSE DETONATION ENGINE PERFORMANCE IN FLIGHT CONDITIONS}

The FTP method has been applied for simulating PDE operation in supersonic flight conditions and estimating its thrust performance. Figure 10 shows 


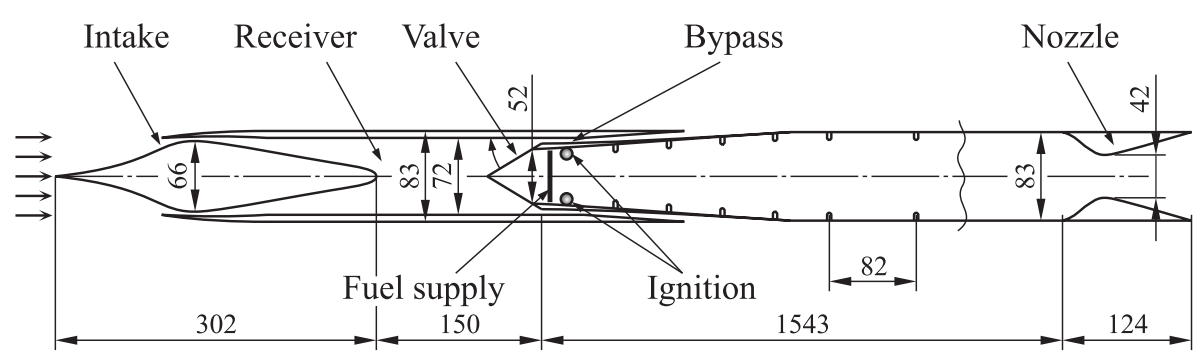

Figure 10 Flying PDE-based vehicle. Dimensions are in millimeters

Table 4 Main parameters and results of calculations

\begin{tabular}{lcc}
\hline Parameter & \multicolumn{2}{c}{ Value } \\
\hline$Z, \mathrm{~km}$ & 9.3 & 16 \\
$P_{a}, \mathrm{MPa}$ & 0.029 & 0.010 \\
$T_{a}, \mathrm{~K}$ & 228 & 216.7 \\
$\tau_{\text {cycle }}, \mathrm{ms}$ & 21 & 21 \\
$f, \mathrm{~Hz}$ & 48 & 48 \\
$P_{*}, \mathrm{MPa}$ & 0.331 & 0.175 \\
$F, \mathrm{~N}$ & 2.05 & 0.77 \\
$F_{R, 1}, \mathrm{~N}$ & 8.21 & 4.54 \\
$F_{R, 2}, \mathrm{~N}$ & 9.25 & 4.52 \\
$\dot{m}_{f}, \mathrm{~kg} / \mathrm{s}$ & 0.00061 & 0.00032 \\
$I_{\mathrm{sp}, 1}, \mathrm{~s}$ & 1720 & 1690 \\
$I_{\mathrm{sp}, 2}, \mathrm{~s}$ & 1890 & 1680 \\
\hline
\end{tabular}

a schematic of the PDE-based axisymmetric vehicle with supersonic air intake, bypass channel, mechanical valve, and nozzle, flying at $\mathrm{M}_{\infty}=3.0$ at the altitude $Z=9.3$ or $16 \mathrm{~km}$. Table 4, among other parameters, shows the static temperature $T_{a}$ and pressure $P_{a}$ of atmospheric air at these altitudes.

The specific geometrical dimensions of the vehicle under consideration are also given in Fig. 10. The total length of the vehicle is $2120 \mathrm{~mm}$, the outer diameter is $83 \mathrm{~mm}$. The detonation chamber consists of two parts, an expanding conical section and a cylindrical section $83 \mathrm{~mm}$ in diameter with regular obstacles in the form of orifice plates (BR $=0.3)$. The pitch of the obstacles in the sec-

tions is 50 and $82 \mathrm{~mm}$, respectively. The last obstacle is mounted at a distance of $510 \mathrm{~mm}$ from the igniter. Downstream of this obstacle, the cylindrical tube $1000 \mathrm{~mm}$ long is smooth-walled. A supersonic nozzle with the throat $42 \mathrm{~mm}$ in diameter is attached to the right end of the detonation chamber. Due to the specific shape of the bypass channel entrance, the mechanical valve blocks $73 \%$ of the engine-duct cross section when the entrance to the detonation chamber is closed and $48 \%$ when it is open.

The engine was assumed to be fueled with gaseous propane. In the calculations, propane was supplied to the flow through the computational cells ("feed" cells) in a chosen cross section of the detonation chamber (shown as a thick vertical line in Fig. 10) by making a provision for a proper mass source in the cells. The chosen value of the mass source ensured the stoichiometric mixture composition in the "feed" cells. Ignition was triggered in the wake of the first orifice plate as shown in Fig. 10. The ignition procedure was the same as described above for the validation tests of the coupled FTP method. The ignition energy 


\section{$\mathrm{C}_{3} \mathrm{H}_{8}$ mass fraction

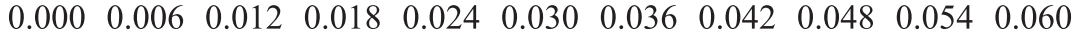

(a)

Temperature / K

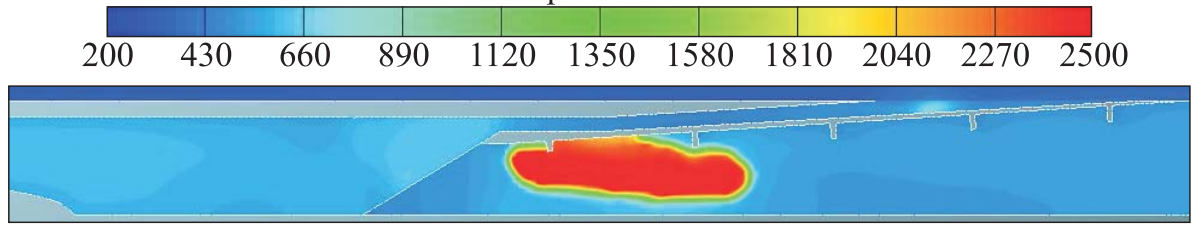

(b)

Temperature / K

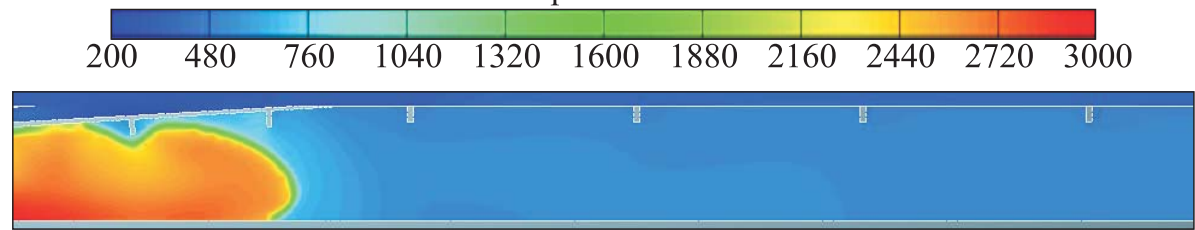

(c)

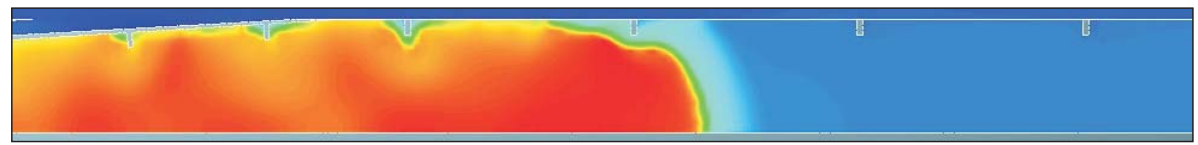

(d)

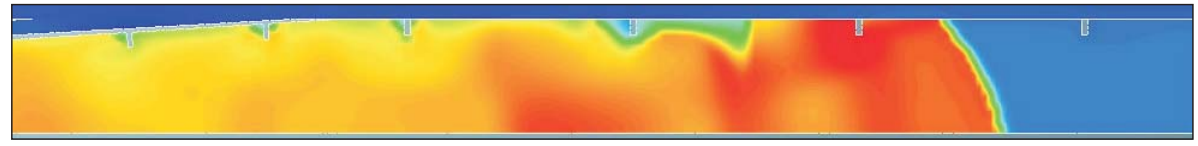

(e)

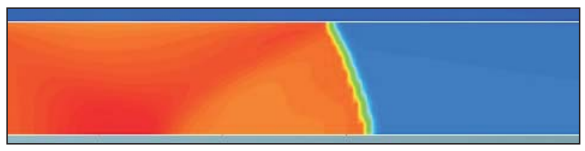

$(f)$

Figure 11 Flow patterns at detonation chamber filling with fuel (a); flame propagation shortly after ignition $(b)$ and at a later stage, when flame accelerates considerably $(c)$; as well as shortly prior to DDT $(d)$ and shortly after DDT $(e)$, and at detonation propagation in the detonation chamber $(f)$. (Refer Frolov and Ivanov, p. 549.) 
Temperature / K

200 \begin{tabular}{rrrrrrrrrrr}
\hline & 510 & 820 & 1130 & 1440 & 1750 & 2060 & 2370 & 2680 & 2990 & 3300
\end{tabular}
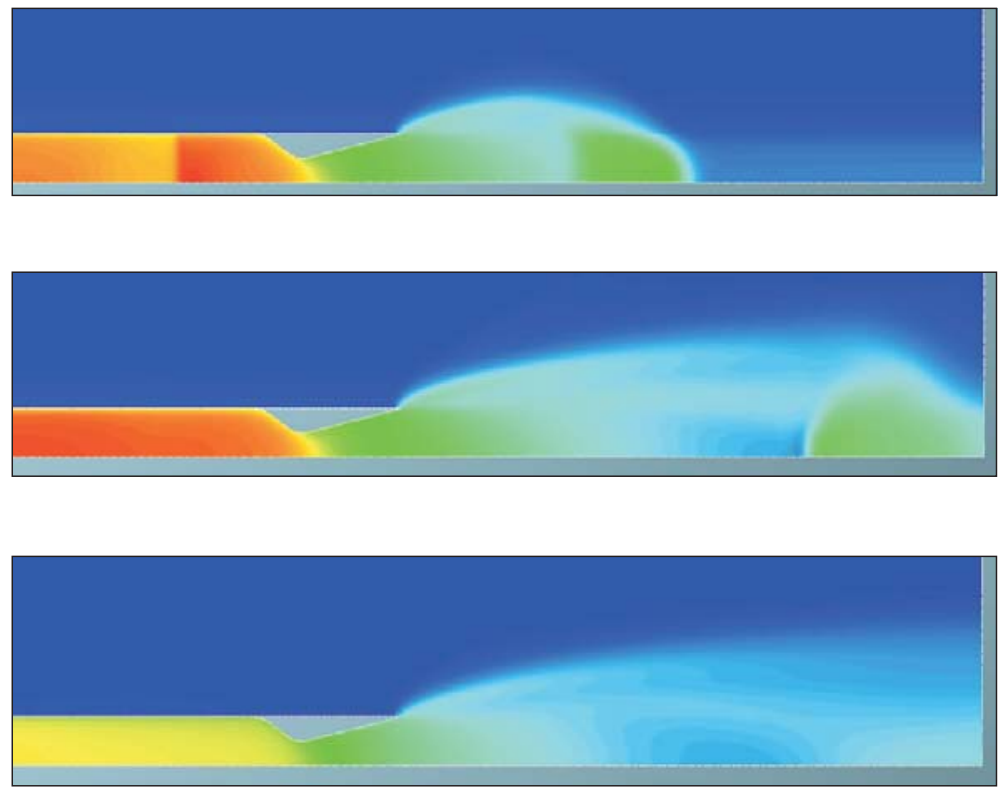

Figure 12 Flow patterns in the nozzle of the PDE-based vehicle and in the exhaust plume. (Refer Frolov and Ivanov, p. 550.)

was on the order of $1 \mathrm{~mJ}$. The cyclic operation process of the PDE included three stages.

At the first stage, when the valve was open, the combustion chamber was getting filled with fuel-air mixture (Fig. 11a). To avoid the contact of the fresh mixture with the combustion products of the previous cycle, the fuel was supplied to the air stream with a certain time delay after valve opening.

When the combustion chamber was filled with the mixture, the valve was closed (instantaneously) and the second stage of the operation process started. After mixture ignition, the flame propagated with acceleration in the turbulent flow of fresh mixture (Figs. $11 b$ to $11 d$ ) and transitioned to a detonation (Fig. 11e). The arising detonation wave propagated further downstream in the combustion chamber (Fig. 11f), traversed the nozzle and escaped into the atmosphere (Fig. 12). Despite the reflection of the detonation wave from the converging nozzle section temporarily produced a negative thrust, the supersonic nozzle was needed to provide a necessary level of backpressure in the detonation chamber. As a result, the reactive mixture ahead of the propagating detonation exhibited an enhanced detonability due to elevated pressure and temperature as well as high turbulence level. 


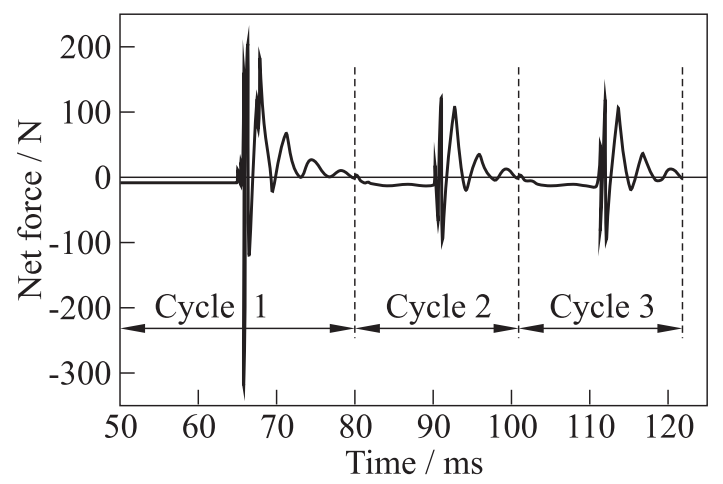

Figure 13 Predicted time history of PDE thrust in the course of three engine cycles

The third stage was the exhaust of combustion products. This stage lasted until the pressure at the valve from the side of combustion chamber dropped to a certain preset value $P_{*}$, which ensured a positive thrust. Thereafter, the valve was open (instantaneously) and the operation cycle repeated.

Figure 13 shows the predicted time history of the net force acting on all surfaces of the PDE-based vehicle in the course of three engine cycles in the flight at $Z=9.3 \mathrm{~km}$. As follows from Fig. 13, the first cycle differs considerably from the subsequent cycles, whereas the second and third cycles are nearly identical. Thus, the operation process becomes reproducible (attains the limit cycle conditions) after the second or third cycle and engine thrust performance can be evaluated starting from the second or third cycle. The first cycle is different from the subsequent cycles due to the difference in the initial conditions for these cycles. Actually, the duration of the first cycle is 30 rather than $21 \mathrm{~ms}$ characteristic for the second and third cycles.

One can readily calculate the cycle-by-cycle total impulse as the area under the curve in Fig. 13. In the second (and third) cycle, the total impulse is equal to $0.043 \mathrm{~N} \cdot \mathrm{s}$. Taking into account that cycle duration is $21 \mathrm{~ms}$, one obtains the net force acting on the PDE vehicle: $F=0.043 / 0.021=2.05$ N. Note that this force is the difference between thrust $F_{T}$ and aerodynamic drag force $F_{R}$, i. e., $F=F_{T}-F_{R}$. Since the net force $F$ is positive, the PDE vehicle under consideration should accelerate at the chosen conditions.

To determine the engine thrust, one has to calculate the drag force $F_{R}$. The drag force can be evaluated by considering the flow in the PDE duct without ignition and combustion, other conditions being equal. By other words, the drag force completely manifests itself in case of engine misfiring. There are two ways to estimate the drag force: with one misfiring event followed by normal operation, and with several successive misfiring events. In the former case, after valve opening the fresh mixture fills the combustion chamber displacing the residual 
hot combustion products from the regular (with ignition) previous cycle and then leaves the engine without ignition. In the latter case, after valve opening the fresh mixture fills the combustion chamber displacing the residual cold unburned mixture from the irregular (with misfire) previous cycle and then leaves the engine without ignition. The net impulse of the drag force was estimated as 0.17246 and $0.19433 \mathrm{~N} \cdot \mathrm{s}$, respectively. Taking into account that $\tau_{\text {cycle }}=21 \mathrm{~ms}$, one obtains $F_{R, 1} \approx-0.17246 / 0.021=-8.21 \mathrm{~N}$ and $F_{R, 2} \approx-0.19433 / 0.021=-9.25 \mathrm{~N}$ where indices 1 and 2 correspond to the first and second way of estimating $F_{R}$. Then, the PDE thrust can be estimated as $F_{T, 1}=F+F_{R, 1} \approx 2.05+8.21=10.26 \mathrm{~N}$ and $F_{T, 2}=F+F_{R, 2} \approx 2.05+9.25=11.3 \mathrm{~N}$.

Based on the values of engine thrust $F_{T}$ and mass flow rate of fuel per single operation cycle $\dot{m}_{f}$, one can estimate the specific impulse of the PDE:

$$
I_{\mathrm{sp}}=\frac{F_{T}}{\dot{m}_{f} g}
$$

where $g$ is the acceleration of gravity. Taking into account that the mass of propane supplied to the combustion chamber in the second cycle was $1.28 \cdot 10^{-5} \mathrm{~kg}$ and $\tau_{\text {cycle }}=21 \mathrm{~ms}$ (operation frequency $f=48 \mathrm{~Hz}$ ), one obtains:

$$
\begin{aligned}
& I_{\mathrm{sp}, 1}=\frac{10.26 \cdot 0.021}{1.28 \cdot 10^{-5} \cdot 9.8} \approx 1720 \mathrm{~s} ; \\
& I_{\mathrm{sp}, 2}=\frac{11.3 \cdot 0.021}{1.28 \cdot 10^{-5} \cdot 9.8} \approx 1890 \mathrm{~s} .
\end{aligned}
$$

Thus, the fuel-based specific impulse of PDE in the conditions of flight at $\mathrm{M}=3.0$ and $Z=9.3 \mathrm{~km}$ is $I_{\mathrm{sp}} \approx 1800 \pm 100 \mathrm{~s}$.

Similar calculations were performed for the flight conditions of the same PDE-based vehicle at $\mathrm{M}=3.0$ and $Z=16 \mathrm{~km}$. All results of calculations are presented in Table 1. It follows from the table that for the flight at $Z=16 \mathrm{~km}$, $I_{\mathrm{sp}, 1} \approx I_{\mathrm{sp}, 2} \approx 1700 \mathrm{~s}$.

\section{CONCLUDING REMARKS}

A coupled FTP method combined with the look-up tables of laminar flame velocities and fuel oxidation has been developed and implemented into the CFD code. The method avoids numerical diffusion of scalar values through the flame front and provides spatial and temporal resolution of preflame self-ignition sites. The algorithms have been tested for several 2D configurations with flame acceleration in smooth-walled and obstructed channels of different length and with the DDT in a channel with regular obstacles and demonstrated good solution convergence and stability. The numerical simulation of the PDE operating on 
DDT in stoichiometric propane-air mixture in conditions of Mach 3.0 flight at the altitude of 9.3 and $16 \mathrm{~km}$ made it possible to estimate the fuel-based specific impulse on the level of 1700-1800 s. This value is higher than the value typical for hydrocarbon-fueled ramjets (1200-1500 s) at these conditions. The DDT run-up distance and time in such conditions were shown to be so short that the engine integrating the supersonic intake, detonation chamber, bypass channel, valve distribution system, and supersonic nozzle could be quite compact with the total length of about $2 \mathrm{~m}$ and could operate at the altitude of 9.3 and $16 \mathrm{~km}$ with positive thrust at a frequency of about $50 \mathrm{~Hz}$ and very low detonation ignition energy on the order of $1 \mathrm{~mJ}$.

The possibility of obtaining a repeatable DDT in hydrocarbon fuel (aviation kerosene)-air mixture with such short run-up distances and times has been recently substantiated experimentally at the SICP [21]. Several engine designs have been suggested and PDE demonstrators fabricated and tested. It has been proved experimentally that DDT in heterogeneous aviation kerosene-air mixture at atmospheric initial pressure can be repeatedly obtained at run-up distances and times shorter than $2 \mathrm{~m}$ and 5-7 ms, respectively, whereas the ignition energy can be as low as $0.1 \mathrm{~J}$. These findings indicate that at zero flight speed conditions, a kerosene-fueled PDE operating on DDT can be fired at frequencies up to 50$60 \mathrm{~Hz}$ provided it is equipped with a starting device for air supply (e.g., fan, compressor, pressurized air bottle, etc.). At flight conditions, ram compression of approach air stream in the engine intake will facilitate filling and purging processes in the detonation chamber as well as decrease the DDT run-up distance and time (due to elevated mixture density), thus increasing the maximum PDE operation frequency.

\section{ACKNOWLEDGMENTS}

The authors thank Prof. Basevich V. Ya., Dr. Belyaev A. A., and Dr. Frolov F. S. for developing look-up tables. This work was supported by the Russian Foundation for Basic Research (grant 11-08-01297-a).

\section{REFERENCES}

1. Lee, J. H. S. 2008. The detonation phenomenon. Cambridge Univ. Press.

2. Bone, W. A., and R. P. Fraser. 1929. A photographic investigation of flame movements in carbonic oxide-oxygen explosions. Philos. Trans. R. Soc. L. A 228:197234.

3. Zel'dovich, Ya. B. 1944. Theory of combustion and detonation of gases. Moscow: USSR Acad. Sci. Publ. 
4. Shchelkin, K. I. 1949. Fast combustion and spin detonation of gases. Moscow: Voenizdat Publ.

5. Oppenheim, A.K. 1972. Introduction to gasdynamics of explosions. Wien-N.Y.: Springer.

6. Lee, J.H.S., and I. Moen. 1980. The mechans of transition from deflagration to detonation in vapor cloud explosions. Prog. Energy Combust. Sci. 6(4):359-89.

7. Higgins, A. J., P. Pinard, A. K. Yoshinaka, and J. H. S. Lee. 2006. In: Pulse detonation engines. Ed. S. M. Frolov. Moscow: TORUS PRESS. 65-86. (In Russian.)

8. Peraldi, O., R. Knystautas, and J.H. S. Lee. 1986. Criteria for transition to detonation in tubes. Proc. Combust. Inst. 21:1629-37.

9. Oran, E., and V. Gamezo. 2007. Origins of the deflagration-to-detonation transition in gas-phase combustion. Combust. Flame 148(1-2):4-47.

10. Gamezo, V., T. Ogawa, and E. Oran. 2008. Flame accelecation and DDT in channels with obstacles: Effect of obstacle spacing. Combust. Flame 155(1-2):302-15.

11. Spalding, D. B. 1976. Mathematical models of turbulent flames: A review. Combust. Sci. Technol. 13:3.

12. Pope, S. B. 1990. Computations of turbulent combustion progress and challenges. Proc. Combust. Inst. 23:591-612.

13. Peters, N. 1986. Laminar flamelet concepts in turbulent combustion. Proc. Combust. Inst. 21:1231-50.

14. Shchelkin, K.I., and Ya. K. Troshin. 1963. Gasdynamics of combustion. Moscow: USSR Acad. Sci. Publ.

15. Frolov, S. M., V. Ya. Basevich, M. G. Neuhaus, and R. Tatschl. 1997. A joint velocity-scalar PDF method for modeling premixed and nonpremixed combustion. In: Advanced computation and analysis of combustion. Eds. G. D. Roy, S. M. Frolov, and P. Givi. Moscow: ENAS Publ. 537-61.

16. Pope, S. B. 1985. PDF methods for turbulent reactive flows. Prog. Energy Combust. Sci. 11(2):119-92.

17. Basevich, V. Ya., and S. M. Frolov. 2006. Overall kintetic mechanisms for modeling multistage self-ignition of hydrocarbons in reacting flows. Rus. J. Chem. Phys. 25(6):54-62.

18. Kerampran, S., D. Desbordes, and B. Veyssiere. 2003. Influence of the pressure waves generated at the initial stage of flame propagation on the DDT process in smooth tubes. In: Confined detonations and pulse detonation engines. Eds. G. Roy, S. Frolov, W. Santoro, and S. Tsyganov. Moscow: TORUS PRESS. 3-16.

19. Frolov, S. M., V. Ya. Basevich, A. A. Belyaev, V.S. Posvyanskii, and Yu. B. Radvogin. 2001. Modeling of confined flame srabilization by bluff bodies. In: Advances in chemical propulsion: Science to technology. Ed. G. D. Roy. Washington, D.C.: CRC Press. 191-214.

20. Ciccarelli, G., C. J. Fowler, and M. Bardon. 2003. Effects of obstacle size and spacing on the initial stage of flame acceleration in an obstacle laden tube. 19th ICDERS Proceedings. Hakone, Japan.

21. Frolov, S. M. 2010. Pulsed detonation propulsion. In: RTO-AVT-VKI Lecture Series "High-Speed Propulsion: Engine Desighn — Integration and Thermal Management". Von Karman Institute for Fluid Dynamics. Paper No. RTO-EN-AVT-185. 